\title{
LIMIT CYCLES AND INVARIANT CYLINDERS FOR A CLASS OF CONTINUOUS AND DISCONTINUOUS VECTOR FIELD IN DIMENTION $2 n$
}

\author{
MAURICIO FIRMINO SILVA LIMA ${ }^{1}$ AND JAUME LLIBRE ${ }^{2}$
}

\begin{abstract}
The subject of this paper concerns with the bifurcation of limit cycles and invariant cylinders from a global center of a linear differential system in dimension $2 n$ perturbed inside a class of continuous and discontinuous piecewise linear differential systems. Our main results show that at most one limit cycle and at most one invariant cylinder can bifurcate using the expansion of the displacement function up to first order with respect to a small parameter. This upper bound is reached. For proving these results we use the averaging theory in a form where the differentiability of the system is not needed.
\end{abstract}

\section{INTRODUCTION}

In control theory and in the study of electrical circuits appear in a natural way the continuous and discontinuous piecewise linear differential systems, see for instance [6], [9] and the references therein. Such differential systems can exhibit complicate dynamics such as those exhibited by general nonlinear differential systems. The limit cycles and the invariant sets by the flow are some of the main components in the qualitative description of the dynamical behavior of a differential system.

In this paper we study the existence of limit cycles and invariant generalized cylinders for the class of control systems represented by

$$
\dot{x}=A_{0} x+\varepsilon F(x)
$$

where

(i) $A_{0} \in \mathcal{M}_{2 n}(\mathbb{R})$ with eigenvalues $\left\{ \pm i p_{1} / q_{1}, \ldots \pm i p_{n} / q_{n}\right\}$ where $p_{k}$ and $q_{k}$ are positive integers for $k=1, \ldots n$ and $\left(p_{k}, q_{k}\right)=1$, where $(.,$.$) denotes$ the greatest common divisor of $p_{k}$ and $q_{k}$.

(ii) $p_{k} / q_{k} \neq p_{l} / q_{l}$ for $k \neq l$;

(iii) $\varepsilon \neq 0$ is a sufficiently small real parameter;

(iv) $F: \mathbb{R}^{2 n} \rightarrow \mathbb{R}^{2 n}$ given by

$$
F(x)=A x+\varphi_{0}\left(k^{T} x\right) b,
$$

with $A \in \mathcal{M}_{2 n}(\mathbb{R})$ and $\varphi_{0}: \mathbb{R} \rightarrow \mathbb{R}$ is the discontinuous function

$$
\varphi_{0}(y)=\left\{\begin{array}{cl}
-1 & y \in(-\infty, 0) \\
1 & y \in(0, \infty)
\end{array}\right.
$$

Key words and phrases. equilibrium point, periodic orbit, limit cycle, invariant cylinder, continuous piecewise linear vector fields, discontinuous piecewise linear vector fields. 
Here the dot denotes derivative with respect to $t$.

Let $Q$ be the least common multiple of $q_{1}, \ldots, q_{n}$. Doing a linear change of variables and a rescaling of the independent variable $t$ we write the matrix $A_{0}$ in its real Jordan normal form with eigenvalues $\left\{ \pm i, \pm i a_{2}, \ldots, \pm i a_{n}\right\}$, where $a_{k}=\alpha_{k} / q$ with $\alpha_{k}=\frac{p_{k} Q}{q_{k}}, k=\{2, \ldots, n\}$ and $q=\frac{p_{1} Q}{q_{1}}$ are positive integers. Note that by (ii) $a_{k} \neq a_{l}$ if $k \neq l$. This $q$ will be used later on in this paper. So $A_{0}$ is

$$
A_{0}=\left(\begin{array}{cccccccc}
0 & -1 & & & & & & \\
1 & 0 & & & & & & \\
& & 0 & -a_{2} & & & & \\
& & a_{2} & 0 & & & & \\
& & & & & & & \\
& & & \ddots & & & & \\
& & & 0 & -a_{n-1} & & \\
& & & a_{n-1} & 0 & & \\
& & & & & 0 & -a_{n} \\
& & & & & a_{n} & 0
\end{array}\right) \text {, }
$$

where the empty entries of the matrix are zeros.

For our purpose we first study the perturbed problem

$$
\dot{x}=A_{0} x+\varepsilon F_{w}(x),
$$

where $F_{w}$ is the piecewise linear function $F_{w}(x)=A x+\varphi_{w}\left(k^{T} x\right) b$ where $\varphi_{w}: \mathbb{R} \rightarrow \mathbb{R}$ is given by

$$
\varphi_{w}(y)=\left\{\begin{array}{cl}
-1 & y \in(-\infty,-w], \\
\frac{y}{w} & y \in(-w, w), \\
1 & y \in[w, \infty) .
\end{array}\right.
$$

Observe that we have two different limit differential systems. More precisely

(i) For $\varepsilon=0$ system (5) becomes

(7)

$$
\begin{aligned}
& \dot{x}_{1}=-x_{2}, \\
& \dot{x}_{2}=x_{1}, \\
& \dot{x}_{3}=-a_{2} x_{4}, \\
& \dot{x}_{4}=a_{2} x_{3}, \\
& \quad \vdots \\
& \dot{x}_{2 n-1}=-a_{n} x_{2 n}, \\
& \dot{x}_{2 n}=a_{n} x_{2 n-1},
\end{aligned}
$$

where the origin is a global center.

(ii) Taking $w \rightarrow 0$ in (5) we obtain the discontinuous two piecewise linear differential system (1).

A limit cycle of a differential system is an isolated periodic orbit in the set of all periodic orbits of the system.

Our main results are the following.

Theorem 1. For every integer $n \geq 2$ at most one limit cycle of the piecewise linear differential system (5) bifurcates from the periodic orbits of system (7), up to first-order expansion of the displacement function of (5) with respect to the small parameter $\varepsilon$. Moreover there are systems (5) having exactly one limit cycle. 
Corollary 2. For every integer $n \geq 2$ at most one limit cycle of the discontinuous piecewise linear differential system (1) bifurcates from the periodic orbits of system (7), up to first-order expansion of the displacement function of (1) with respect to the small parameter $\varepsilon$. Moreover there are systems (1) having exactly one limit cycle.

Theorem 3. For every integer $n \geq 2$ at most one invariant cylinder homeomorphic to $\mathbb{R} \times\left(\mathbb{S}^{1}\right)^{n-1}$ of the piecewise linear differential system (5) bifurcates from (7), up to first-order expansion of the displacement function of (5) with respect to the small parameter $\varepsilon$. Moreover there are systems (5) having exactly one invariant cylinder. This cylinder is fulfilled of periodic orbits.

Corollary 4. For every integer $n \geq 2$ at most one invariant cylinder homeomorphic to $\mathbb{R} \times\left(\mathbb{S}^{1}\right)^{n-1}$ of the discontinuous piecewise linear differential system 1 bifurcates from (7), up to first-order expansion of the displacement function of (1) with respect to the small parameter $\varepsilon$. Moreover there are systems (1) having exactly one invariant cylinder. This cylinder is fulfilled of periodic orbits.

Theorem 1 is an extension first of the main results of [4] where the case $\alpha_{j}=1$ for $j=1, \cdots, n$ with $A_{0}$ semi-simple is considered, and second of the main results of [7] where the case $1: m$ with integer $m>1$ in dimension 4 is studied.

This paper is organized as follows. In section 2 we present the averaging theory of first order in the form obtained in [1] for detecting limit cycles and invariant cylinders. The advantage of this result is that the smoothness assumptions for the vector field of the differential system it is not necessary. In particular, it can be applied to piecewise linear differential systems, which are not $C^{2}$ (not even $C^{1}$ ), as it was required in its classical version, see for instance, Theorem 11.5 of [10]. This version of averaging theory has been used in some previous papers, see for instance, [2], [4] and [5]. We note that when the averaged function of first order is not identically zero (and this will be the case in this paper), it is sufficient for studying the periodic orbits of the perturbed system. For more details see section 2 and $[1]$.

In section 3 we prove Theorem 1. For this purpose first we prove Lemma 8 where we reduce the number of parameters by a linear change of coordinates. In the next step we put the system in the standard form for applying averaging, this is done in Lemma 14.

In section 4 we prove Theorem 3 which needs Lemma 20 where a change of coordinates is presented for writing the system in the normal form of the averaging theory. In section 5 we give an illustrative example where a discontinuous piecewise linear differential system exhibits a unique limit cycle and a unique invariant cylinder.

\section{First-Order Averaging Theory}

The aim of this section is to present the first-order averaging theory as obtained in [1]. In this version differentiability of the vector field is not needed. The specific conditions for the existence of a simple isolated zero of the averaged function are given in terms of the Brouwer degree. In fact, the Brouwer degree theory is the key point in the proof of this theorem. We remind here that continuity of some finite dimensional function is a sufficient condition for the existence of its Brouwer degree (see [8] for precise definitions). 
Theorem 5. We consider the following differential system

$$
\dot{x}=\varepsilon H(t, x)+\varepsilon^{2} R(t, x, \varepsilon),
$$

where $H: \mathbb{R} \times D \rightarrow \mathbb{R}^{n}, R: \mathbb{R} \times D \times\left(-\varepsilon_{f}, \varepsilon_{f}\right) \rightarrow \mathbb{R}^{n}$ are continuous functions, $T$ periodic in the first variable, and $D$ is an open subset of $\mathbb{R}^{n}$. We define $h: D \rightarrow \mathbb{R}^{n}$ as

$$
h(z)=\int_{0}^{T} H(s, z) d s,
$$

and assume that:

(i) $H$ and $R$ are locally Lipschitz with respect to $x$;

(ii) for $a \in D$ with $h(a)=0$, there exists a neighborhood $V$ of a such that $h(z) \neq 0$ for all $z \in \bar{V} \backslash\{a\}$ and $d_{B}(h, V, a) \neq 0$ (here $d_{B}(h, V, a)$ denotes the Brouwer degree of $h$ at a).

Then, for $|\varepsilon|>0$ sufficiently small, there exists an isolated T-periodic solution $\psi(., \varepsilon)$ of system (8) such that $\psi(0, \varepsilon) \rightarrow a$ as $\varepsilon \rightarrow 0$.

Here we will need some facts from the proof of Theorem 5. Hypothesis (i) assures the existence and uniqueness of the solution of each initial value problem on the interval $[0, T]$. Hence, for each $z \in D$, it is possible to denote by $x(., z, \varepsilon)$ the solution of $(8)$ with the initial value $x(0, z, \varepsilon)=z$. We consider also the displacement function $\zeta: D \times\left(-\varepsilon_{f}, \varepsilon_{f}\right) \rightarrow \mathbb{R}^{n}$ defined by

$$
\zeta(z, \varepsilon)=\int_{0}^{T}\left[\varepsilon H(t, x(t, z, \varepsilon))+\varepsilon^{2} R(t, x(t, z, \varepsilon), \varepsilon)\right] d t .
$$

From the proof of Theorem 5 we extract the following facts.

Remark 6. For every $z \in D$ the following relation holds

$$
x(T, z, \varepsilon)-x(0, z, \varepsilon)=\zeta(z, \varepsilon) .
$$

The function $\zeta$ can be written in the form

$$
\zeta(z, \varepsilon)=\varepsilon h(z)+O\left(\varepsilon^{2}\right),
$$

where $h$ is given by (9) and the symbol $O\left(\varepsilon^{2}\right)$ denotes a bounded function on every compact subset of $D \times\left(-\varepsilon_{f}, \varepsilon_{f}\right)$ and of order $\varepsilon^{2}$. Moreover, for $|\varepsilon|$ sufficiently small, $z=\psi(0, \varepsilon)$ is a isolated zero of $\zeta(., \varepsilon)$.

Note that from Remark 6 it follows that a zero of the displacement function $\zeta(z, \varepsilon)$ at time $T$ provides initial conditions for a periodic orbit of the system of period $T$. We also remark that $h(z)$ is the displacement function up to terms of order $\varepsilon$. Consequently the zeros of $h(z)$, when $h(z)$ is not identically zero, also provides periodic orbits of period $T$.

For a given system there is the possibility that the function $\zeta$ is not globally differentiable, but the function $h$ is $C^{1}$ as we shall see in Section 3 and 4. In fact, only differentiability in some neighborhood of a fixed isolated zero of $h$ could be enough. When this is the case, one can use the following remark in order to verify the hypothesis (ii) of Theorem 5 .

Remark 7. Let $h: D \rightarrow \mathbb{R}^{n}$ be a $C^{1}$ function, with $h(a)=0$, where $D$ is an open subset of $\mathbb{R}^{n}$ and $a \in D$. Whenever $a$ is a simple zero of $h$ (i.e. the Jacobian $J_{h}(a)$ of $h$ at $a$ is not zero), then there exists a neighborhood $V$ of a such that $h(z) \neq 0$ for all $z \in \bar{V} \backslash\{a\}$ and $d_{B}(h, V, a) \in\{-1,1\}$. 
The averaging theory provides information on the location of the periodic orbits. Thus if $a$ is a zero of the averaged function $h(z)$ with $d_{B}(f, V, a) \neq 0$, then there is a limit cycle $\varphi(t, \varepsilon)$ of system (8) satisfying that $\varphi(0, \varepsilon) \rightarrow a$ when $\varepsilon \rightarrow 0$. So $a$ is an initial condition for the periodic orbit which bifurcates to the limit cycle $\varphi(t, \varepsilon)$.

The averaging theory for $C^{2}$ differential system also provides in the hyperbolic case the stability or unstability of the periodic orbits. For discontinuous differential systems a weaker result on the stability and unstability can be found in [3].

\section{Proof of Theorem 1}

As before we will assume that $A_{0}$ is given by its real Jordan normal form (4). Moreover a set of lemmas will be presented to put the system (5) in a more tractable way for using the averaging method.

The next lemma writes system (5) to a convenient normal form.

Lemma 8. If the vector $k=\left(k_{1}, k_{2}, \ldots, k_{2 n-1}, k_{2 n}\right)$ is such that $\prod_{j=1}^{n}\left(k_{2 j-1}^{2}+\right.$ $\left.k_{2 j}^{2}\right) \neq 0$, then there exists a linear change of coordinates that sending the system (5) to the system

$$
\dot{x}=A_{1} x+\varepsilon \bar{A} x+\varepsilon \varphi_{w}\left(x_{1}\right) \bar{b},
$$

where $\bar{A} \in \mathcal{M}_{2 n}(\mathbb{R})$ and $\bar{b} \in \mathbb{R}^{2 n}$ are functions of $A$ and $b$. Moreover

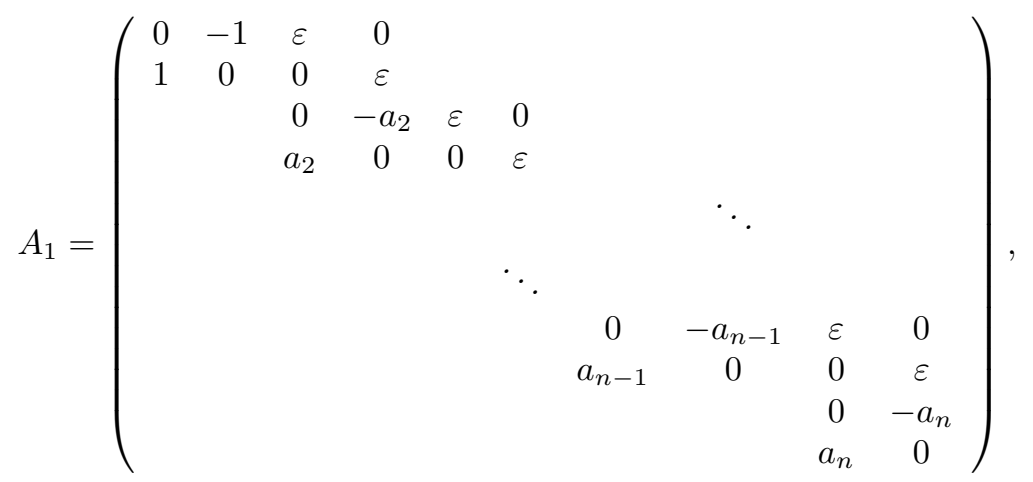

where all the empty entries of $A_{1}$ are zero.

Proof. To prove the lemma we must find $J$ satisfying

$$
\begin{gathered}
J^{-1} A_{0} J=A_{1}, \\
k^{T} J=e_{1}^{T} .
\end{gathered}
$$

The proof will be done by induction on $n$.

If $n=2$ the vector field is in a 4-dimensional space and a straightforward calculation shows that the matrix

$$
J_{4}=\left(\begin{array}{ccccc}
\frac{k_{1}}{k_{1}^{2}+k_{2}^{2}} & -\frac{k_{2}}{k_{1}^{2}+k_{2}^{2}} & -\frac{\varepsilon k_{2}}{\left(k_{1}^{2}+k_{2}^{2}\right)\left(a_{2}-1\right)} & -\frac{\varepsilon k_{1}}{\left(k_{1}^{2}+k_{2}^{2}\right)\left(a_{2}-1\right)} \\
\frac{k_{2}}{k_{1}^{2}+k_{2}^{2}} & \frac{k_{1}}{k_{1}^{2}+k_{2}^{2}} & \frac{\varepsilon k_{2}}{\left(k_{1}^{2}+k_{2}^{2}\right)\left(a_{2}-1\right)} & -\frac{\varepsilon k_{4}}{\left(k_{1}^{2}+k_{2}^{2}\right)\left(a_{2}-1\right)} \\
0 & 0 & \frac{\varepsilon k_{3}}{\left(k_{3}^{2}+k_{4}^{2}\right)\left(a_{2}-1\right)} & \frac{\varepsilon k_{3}}{\left(k_{3}^{2}+k_{4}^{2}\right)\left(a_{2}-1\right)} \\
0 & 0 & -\frac{\varepsilon k_{4}}{\left(k_{3}^{2}+k_{4}^{2}\right)\left(a_{2}-1\right)} & \frac{J_{3,2}}{\left(k_{3}^{2}+k_{4}^{2}\right)\left(a_{2}-1\right)}
\end{array}\right)=\left(\begin{array}{cc}
J_{1,1} & J_{1,2} \\
& \\
J_{2,1} & J_{2,2}
\end{array}\right)
$$

satisfies system (12), where the matrix $J_{i, j} \in \mathcal{M}_{2}(\mathbb{R})$ and $J_{2,1}=0$. Note that, in this case, $J_{4}$ is well defined and invertible because its determinant $\varepsilon^{2} /\left[\left(a_{2}-1\right)^{2}\left(k_{1}^{2}+\right.\right.$ $\left.\left.k_{2}^{2}\right)\left(k_{3}^{2}+k_{4}^{2}\right)\right] \neq 0$ and $a_{2} \neq 1$. 
Suppose that the result is valid up to $n-1$. That is, there exists a $(2 n-$ 2)-invertible matrix $J_{2 n-2}$ of the form

$$
J_{2 n-2}=\left(\begin{array}{ccccc}
J_{1,1}^{*} & J_{1,2}^{*} & \cdots & J_{1, n-2}^{*} & J_{1, n-1}^{*} \\
J_{2,1}^{*} & J_{2,2}^{*} & \cdots & J_{2, n-2}^{*} & J_{2, n-1}^{*} \\
\cdots & \cdots & \cdots & \cdots & \cdots \\
J_{n-2,1}^{*} & J_{n-2,2}^{*} & \cdots & J_{n-2, n-2}^{*} & J_{n-2, n-1}^{*} \\
J_{n-1,1}^{*} & J_{n-1,2}^{*} & \cdots & J_{n-1, n-2}^{*} & J_{n-1, n-1}^{*}
\end{array}\right),
$$

with $J_{i, j}^{*} \in \mathcal{M}_{2}(\mathbb{R})$ and $J_{i, j}^{*}=0$ if $i>j$ and satisfying system (12).

Now we have to find a matrix $J_{2 n}$ of the form

$$
\left.\begin{array}{ccccc}
J_{1,1}^{*} & \cdots & J_{1, n-2}^{*} & \left(\begin{array}{cc}
j_{1,2 n-3}^{*} & j_{1,2 n-2}^{*} \\
j_{2,2 n-3}^{*} & j_{2,2 n-2}^{*}
\end{array}\right) & \left(\begin{array}{cc}
j_{1,2 n-1} & j_{1,2 n} \\
j_{2,2 n-1} & j_{2,2 n}
\end{array}\right) \\
\vdots & \vdots & \vdots \\
J_{n-1,1}^{*} & \cdots & J_{n-1, n-2}^{*} & \left(\begin{array}{cc}
j_{2 n-3,2 n-3}^{*} & j_{2 n-3,2 n-2}^{*} \\
j_{2 n-2,2 n-3}^{*} & j_{2 n-2,2 n-2}^{*}
\end{array}\right) & \left(\begin{array}{cc}
j_{2 n-3,2 n-1} & j_{2 n-3,2 n} \\
j_{2 n-2,2 n-1} & j_{2 n-2,2 n}
\end{array}\right) \\
\left(\begin{array}{cc}
0 & 0 \\
0 & 0
\end{array}\right) & \cdots & \left(\begin{array}{cc}
0 & 0 \\
0 & 0
\end{array}\right) & \left(\begin{array}{cc}
0 \\
0 & 0
\end{array}\right) & \left(\begin{array}{cc}
j_{2 n-1,2 n-1} & j_{2 n-1,2 n} \\
j_{2 n, 2 n-1} & j_{2 n, 2 n}
\end{array}\right)
\end{array}\right)
$$

satisfying system (12) and where the elements with $\left(^{*}\right)$ are fixed by the previous step in the sense that the submatrix with terms $\left(^{*}\right)$ satisfies (12) in dimension $2 n-2$. Moreover $J_{i, j}=0$ if $i>j$.

Some calculations show that the equation $J A_{1}-A_{0} J=0$ is equivalent to the following system of equations:

$$
\begin{array}{ll}
\varepsilon j_{2 k-1,2 n-3}^{*}+a_{n} j_{2 k-1,2 n}+a_{k} j_{2 k, 2 n-1}=0, & k=1, \ldots, n-1, \\
\varepsilon j_{2 k, 2 n-3}^{*}+a_{n} j_{2 k, 2 n}-a_{k} j_{2 k-1,2 n-1}=0, & k=1, \ldots, n-1, \\
\varepsilon j_{2 k-1,2 n-2}^{*}-a_{n} j_{2 k-1,2 n-1}+a_{k} j_{2 k, 2 n}=0, & k=1, \ldots, n-1, \\
\varepsilon j_{2 k, 2 n-2}^{*}-a_{n} j_{2 k, 2 n-1}-a_{k} j_{2 k-1,2 n}=0, & k=1, \ldots, n-1, \\
a_{n} j_{2 n-1,2 n}+a_{n} j_{2 n, 2 n-1}=0, & \\
a_{n} j_{2 n, 2 n}-a_{n} j_{2 n-1,2 n-1}=0 . &
\end{array}
$$

From the first and fourth equations of (13) we obtain

$$
\begin{gathered}
j_{2 k-1,2 n}=\frac{\varepsilon\left(a_{n} j_{2 k-1,2 n-3}^{*}+a_{k} j_{2 k, 2 n-2}^{*}\right)}{a_{k}^{2}-a_{n}^{2}} \text { and } \\
j_{2 k, 2 n-1}=\frac{\varepsilon\left(a_{k} j_{2 k-1,2 n-3}^{*}+a_{n} j_{2 k, 2 n-2}^{*}\right)}{a_{k}^{2}-a_{n}^{2}}
\end{gathered}
$$

for $k=1, \ldots, n-1$ and from the second and third of (13) we obtain

$$
\begin{aligned}
& j_{2 k, 2 n}=\frac{\varepsilon\left(a_{n} j_{2 k, 2 n-3}^{*}-a_{k} j_{2 k-1,2 n-2}^{*}\right)}{a_{k}^{2}-a_{n}^{2}} \text { and } \\
& j_{2 k-1,2 n-1}=\frac{\varepsilon\left(a_{k} j_{2 k, 2 n-3}^{*}-a_{n} j_{2 k-1,2 n-2}^{*}\right)}{a_{k}^{2}-a_{n}^{2}}
\end{aligned}
$$

for $k=1, \ldots, n-1$.

Moreover as $a_{n}^{2}-a_{k}^{2} \neq 0$, for all $k=1, \ldots, n-1$, the solutions found above are unique.

Now from the last two equations of (13) we have

$$
-j_{2 n-1,2 n}=j_{2 n, 2 n-1}=\beta, \quad j_{2 n, 2 n}=j_{2 n-1,2 n-1}=\alpha .
$$


Considering the equation $k^{T} J=e_{1}^{T}$ the previous constants must satisfy

$$
\begin{aligned}
& k_{2 n-1} \alpha+k_{2 n} \beta=-\sum_{i=1}^{2 n-2} k_{i} j_{i, 2 n-1}, \\
& k_{2 n} \alpha-k_{2 n-1} \beta=-\sum_{i=1}^{2 n-2} k_{i} j_{i, 2 n} .
\end{aligned}
$$

This system has a single solution if $k_{2 n-1}^{2}+k_{2 n}^{2} \neq 0$. This completes the proof of the lemma.

The next result will put the system in the standard form for applying the averaging method. This will be done via a convenient change of coordinates.

Lemma 9. Doing the changing of coordinates from $\left(x_{1}, x_{2}, x_{3}, x_{4}, \ldots, x_{2 n-1}, x_{2 n}\right)$ to $\left(r_{1}, \ldots, r_{n}, \theta_{1}, \ldots, \theta_{n}\right)$ given by

$$
\begin{array}{ll}
x_{1}=r_{1} \cos \theta_{1}, & x_{2}=r_{1} \sin \theta_{1}, \\
x_{2 k-1}=r_{k} \cos \left(a_{k} \theta_{1}+\theta_{k}\right), & x_{2 k}=r_{k} \sin \left(a_{k} \theta_{1}+\theta_{k}\right), \quad k=2, \ldots, n,
\end{array}
$$

and considering $\theta_{1}$ as the new time system (11) becomes

$$
\begin{aligned}
& \frac{d r_{1}}{d \theta_{1}}=\varepsilon H_{1}\left(\theta_{1}, r_{1}, \ldots, r_{n}, \theta_{2}, \ldots \theta_{n}\right)+O\left(\varepsilon^{2}\right), \\
& \frac{d r_{k}}{d \theta_{1}}=\varepsilon H_{k}\left(\theta_{1}, r_{1}, \ldots, r_{n}, \theta_{2}, \ldots \theta_{n}\right)+O\left(\varepsilon^{2}\right), \quad k=2, \ldots, n, \\
& \frac{d \theta_{k}}{d \theta_{1}}=\varepsilon G_{k}\left(\theta_{1}, r_{1}, \ldots, r_{n}, \theta_{2}, \ldots \theta_{n}\right)+O\left(\varepsilon^{2}\right), \quad k=2, \ldots, n,
\end{aligned}
$$

where

$$
\begin{aligned}
H_{1}= & r_{2} \cos \left(\left(a_{2}-1\right) \theta_{1}+\theta_{2}\right)+\cos \theta_{1} F_{1}+\sin \theta_{1} F_{2}, \\
H_{k}= & r_{k+1} \cos \left(\left(a_{k+1}-a_{k}\right) \theta_{1}+\left(\theta_{k+1}-\theta_{k}\right)\right)+\cos \left(a_{k} \theta_{1}+\theta_{k}\right) F_{2 k-1} \\
& +\sin \left(a_{k} \theta_{1}+\theta_{k}\right) F_{2 k}, \\
H_{n}= & \cos \left(a_{n} \theta_{1}+\theta_{n}\right) F_{2 n-1}+\sin \left(a_{n} \theta_{1}+\theta_{n}\right) F_{2 n}, \\
G_{k}= & -\frac{a_{k}}{r_{1}}\left[r_{2} \sin \left(\left(a_{2}-1\right) \theta_{1}+\theta_{2}\right)+\cos \theta_{1} F_{2}-\sin \theta_{1} F_{1}\right]+ \\
& \frac{1}{r_{k}}\left[r_{k+1} \sin \left(\left(a_{k+1}-a_{k}\right) \theta_{1}+\left(\theta_{k+1}-\theta_{k}\right)\right)+\cos \left(a_{k} \theta_{1}+\theta_{k}\right) F_{2 k}-\right. \\
& \left.\sin \left(a_{k} \theta_{1}+\theta_{k}\right) F_{2 k-1}\right], \\
G_{n}= & -\frac{a_{n}}{r_{1}}\left[r_{2} \sin \left(\left(a_{2}-1\right) \theta_{1}+\theta_{2}\right)+\cos \theta_{1} F_{2}-\sin \theta_{1} F_{1}\right]+ \\
& \frac{1}{r_{n}}\left[\cos \left(a_{n} \theta_{1}+\theta_{n}\right) F_{2 n}-\sin \left(a_{n} \theta_{1}+\theta_{n}\right) F_{2 n-1}\right],
\end{aligned}
$$

for $H_{k}$ and $G_{k}$ the subindex $k$ varies from 2 to $n-1$. The expressions of $F_{l}$ for $l=1, \cdots, 2 n$ are given in Appendix 1 .

We take $\varepsilon_{f}$ sufficiently small, $m$ arbitrarily large, and $D_{m}=(1 / m, m) \times{ }^{(n)}$. $\times(1 / m, m) \times \mathbb{R} \times \stackrel{(n-1)}{\cdots} \times \mathbb{R}$. Then the vector field of system (14) is well defined and continuous on $\mathbb{R} \times D_{m} \times\left(-\varepsilon_{f}, \varepsilon_{f}\right)$. Moreover it is $2 \pi q$-periodic with respect to $\theta_{1}$ and globally Lipschitz with respect to $\left(r_{1}, \ldots, r_{n}, \theta_{2}, \ldots \theta_{n}\right)$. 
Proof. Considering the variables $\left(\theta_{1}, r_{1}, \ldots, r_{n}, \theta_{2}, \ldots \theta_{n}\right)$ system (11) becomes

$$
\begin{array}{ll}
\dot{\theta_{1}}=1+\frac{\varepsilon}{r_{1}}\left(r_{2} \sin \left(\left(a_{2}-1\right) \theta_{1}+\theta_{2}\right)+\cos \theta_{1} F_{2}-\sin \theta_{1} F_{1}\right), & \\
\dot{r}_{1}=\varepsilon H_{1}\left(\theta_{1}, r_{1}, \ldots, r_{n}, \theta_{2}, \ldots \theta_{n}\right), & k=2, \ldots, n, \\
\dot{r}_{k}=\varepsilon H_{k}\left(\theta_{1}, r_{1}, \ldots, r_{n}, \theta_{2}, \ldots \theta_{n}\right), & k=2, \ldots, n . \\
\dot{\theta}_{k}=\varepsilon G_{k}\left(\theta_{1}, r_{1}, \ldots, r_{n}, \theta_{2}, \ldots \theta_{n}\right), & k \text {. }
\end{array}
$$

Observe that the functions on the right hand of (16) have the form given by (15). So from the fact that $a_{k}=\alpha_{k} / q$ it is easy to see that they are $2 \pi q$-periodic in the variable $\theta_{1}$.

Now for $|\varepsilon|$ sufficiently small we have $\dot{\theta_{1}}(t)>0$ for each $\theta_{1}$ such that $\left(\theta_{1}, r_{1}, \ldots, r_{n}\right.$, $\left.\theta_{2}, \ldots \theta_{n}\right) \in \mathbb{R} \times D_{m}$. A suitable time-rescaling allows to take $\theta_{1}$ as the time and so $(16)$ can be written as a $(2 n-1)$-dimensional system. Expanding this system with respect to $\varepsilon$ in Taylor series we obtain the desired form (14). Moreover the periodicity of the $O\left(\varepsilon^{2}\right)$ functions follows from the fact that these functions are obtained from the previous ones by this expansion in Taylor series. The remaining affirmations follow by direct computations.

In Lemma 9 we have obtained for vector field (5) a form where we are able to apply the averaging theory.

For this purpose we have to find the corresponding averaging function (9).

We call $f: D_{m} \rightarrow \mathbb{R}^{2 n-1}$ the function $f=\left(h_{1}, \ldots, h_{n}, g_{2}, \ldots, g_{n}\right)$ defined by

$$
h_{k}(r, R, s)=\int_{0}^{2 \pi q} H_{k}\left(\theta_{1}, r_{1}, \ldots, r_{n}, \theta_{2}, \ldots \theta_{n}\right) d \theta_{1} \text { for } k=1, \ldots, n
$$

and

$$
g_{l}(r, R, s)=\int_{0}^{2 \pi q} G_{l}\left(\theta_{1}, r_{1}, \ldots, r_{n}, \theta_{2}, \ldots \theta_{n}\right) d \theta_{1} \text { for } l=2, \ldots, n .
$$

In order to compute the averaging function we present below some integral that can be easily calculated

$$
\begin{gathered}
\int_{0}^{2 \pi q} \cos \theta_{1} \sin \theta_{1} d \theta_{1}=0, \\
\int_{0}^{2 \pi q} \cos ^{2}\left(\alpha \theta_{1}+\theta_{k}\right) d \theta_{1}=\pi q=\int_{0}^{2 \pi q} \sin ^{2}\left(\alpha \theta_{1}+\theta_{k}\right) d \theta_{1}, \\
\int_{0}^{2 \pi q} \cos \theta_{1} \sin \left(\alpha \theta_{1}+\theta_{k}\right) d \theta_{1}=0, \quad \forall k=2, \ldots, n \text { and } \alpha \neq 1, \\
\int_{0}^{2 \pi q} \cos \theta_{1} \cos \left(\alpha \theta_{1}+\theta_{k}\right) d \theta_{1}=0, \text { with } k, \alpha \neq 1, \\
\int_{0}^{2 \pi q} \sin \theta_{1} \sin \left(\alpha \theta_{1}+\theta_{k}\right) d \theta_{1}=0, \text { with } k, \alpha \neq 1, \\
\int_{0}^{2 \pi q} \sin \theta_{1} \cos \left(\alpha \theta_{1}+\theta_{k}\right) d \theta_{1}=0, \quad \forall k=2, \ldots, n \text { and } \alpha \neq 1 .
\end{gathered}
$$

Now for each $r_{1}>0$ define

$$
I_{1}\left(r_{1}, w\right)=\int_{0}^{2 \pi q} \varphi_{w}\left(r_{1} \cos \theta_{1}\right) \cos \theta_{1} d \theta_{1}
$$




$$
\begin{gathered}
I_{2}\left(r_{1}, w\right)=\int_{0}^{2 \pi q} \varphi_{w}\left(r_{1} \cos \theta_{1}\right) \sin \theta_{1} d \theta_{1}, \\
I_{2 k-1}\left(r_{1}, w\right)=\int_{0}^{2 \pi q} \varphi_{w}\left(r_{1} \cos \theta_{1}\right) \sin \left(a_{k} \theta_{1}\right) d \theta_{1}, \quad k=2, \ldots, n, \\
I_{2 k}\left(r_{1}, w\right)=\int_{0}^{2 \pi q} \varphi_{w}\left(r_{1} \cos \theta_{1}\right) \cos \left(a_{k} \theta_{1}\right) d \theta_{1}, \quad k=2, \ldots, n,
\end{gathered}
$$

where $\varphi_{w}$ is the real function given in (6).

A direct calculation shows that

$$
I_{2}\left(r_{1}, w\right)=0 \text { and } I_{2 k-1}\left(r_{1}, w\right)=0, \quad \forall r_{1}>0 .
$$

Moreover

$$
I_{1}\left(r_{1}, w\right)=\left\{\begin{array}{cl}
\frac{\pi q r_{1}}{w} & 0<r_{1} \leq w, \\
2 q\left(\frac{r_{1}}{w} \operatorname{arccsc}\left(\frac{r_{1}}{w}\right)+\frac{\sqrt{r_{1}^{2}-w^{2}}}{r_{1}}\right) & r_{1}>w,
\end{array}\right.
$$

and

$$
I_{2 k}\left(r_{1}, w\right)=\left\{\begin{array}{cl}
0 & 0<r_{1} \leq w \\
2 J_{k}\left(r_{1}, w\right) & r_{1}>w
\end{array}\right.
$$

where

$$
\begin{aligned}
J_{k}\left(r_{1}, w\right)= & \left(\frac{1}{a_{k}}-\frac{a_{k}}{a_{k}^{2}-1}\right) \sin \left[a_{k} \arccos \left(\frac{w}{r_{1}}\right)\right]+ \\
& \frac{r_{1} \sin \left[\arccos \left(\frac{w}{r_{1}}\right)\right] \cos \left[a_{k} \arccos \left(\frac{w}{r_{1}}\right)\right]}{w\left(a_{k}^{2}-1\right)}
\end{aligned}
$$

for $k=2, \ldots, n$.

Lemma 10. The system obtained for the averaged function $f$ is given by

$$
\begin{aligned}
h_{1}= & \pi q r_{1}\left(a_{11}+a_{22}\right)+b_{1} I_{1}\left(r_{1}, w\right)=0, \\
h_{k}= & \pi q r_{k}\left(a_{2 k-1,2 k-1}+a_{2 k, 2 k}\right)+\left(b_{2 k-1} \cos \theta_{k}+b_{2 k} \sin \theta_{k}\right) I_{2 k}\left(r_{1}, w\right)=0, \\
g_{k}= & \pi q\left(a_{2 k, 2 k-1}-a_{2 k-1,2 k}+a_{k} a_{2,1}-a_{k} a_{1,2}\right)+ \\
& \frac{1}{r_{1} r_{k}}\left[a_{k} b_{2} r_{k} I_{1}\left(r_{1}, w\right)-r_{1}\left(-b_{2 k} \cos \theta_{k}+b_{2 k-1} \sin \theta_{k}\right)\right] I_{2 k}\left(r_{1}, w\right)=0,
\end{aligned}
$$

where $k=2, \cdots, n$ and $\bar{A}=\left(a_{i j}\right), 1 \leq i, j \leq 2 n$ and $\bar{b}=\left(b_{l}\right), l=1, \ldots, 2 n$ are the ones given in Lemma 8.

Proof. The proof of this lemma is directly obtained using the expression of $H_{1}, H_{k}$ and $G_{k}$ with $k=\{2, \ldots, n\}$ given in Lemma 9 , the previous integrals and the fact that $a_{k} \neq a_{l}$ for $k \neq l$.

Now we can prove Theorem 1. 
Proof of Theorem 1. We have to find the zeroes of system (17). If $0<r_{1} \leq w$ then system (17) becomes

$$
\begin{aligned}
& h_{1}=\pi q r_{1}\left(a_{11}+a_{22}\right)+b_{1} \frac{\pi q r_{1}}{w}=0, \\
& h_{k}=\pi q r_{k}\left(a_{2 k-1,2 k-1}+a_{2 k, 2 k}\right)=0, \\
& g_{k}=\pi q\left(a_{2 k, 2 k-1}-a_{2 k-1,2 k}+a_{k} a_{2,1}-a_{k} a_{1,2}+\frac{a_{k} b_{2}}{w}\right)=0,
\end{aligned}
$$

where $k=2, \cdots, n$.

This system either has no solution, or has a continuum of solutions because the variables $\theta_{k}$ does not appear explicitly in the system. So, in this case, we do not find any limit cycle.

Now if $r_{1}>w$, from the first equation of (17) we have that the function $I_{1}\left(r_{1}, w\right)$ satisfies

(i) For each $w$ fixed $\frac{\partial^{2} I_{1}}{\partial r_{1}^{2}}\left(r_{1}, w\right)=-4 w^{2} q /\left(r_{1}^{2} \sqrt{r_{1}^{2}-w^{2}}\right)<0$ and so the graph of $I_{1}(., w)$ is convex.

(ii) For each $w$ fixed $\frac{\partial I_{1}}{\partial r_{1}}\left(r_{1}, w\right)=\frac{2 q}{w} \operatorname{arccsc}\left(\frac{r_{1}}{w}\right)-2 q \frac{\sqrt{1-\frac{w^{2}}{r_{1}^{2}}}}{r_{1}}$ and we have $\lim _{r \searrow w} \frac{\partial I_{1}}{\partial r_{1}}\left(r_{1}, w\right)=\frac{\pi q}{w}$ and $\lim _{r \rightarrow \infty} \frac{\partial I_{1}}{\partial r_{1}}\left(r_{1}, w\right)=0$. From the fact that $\frac{\partial I_{1}}{\partial r_{1}}$ is decreasing (by (i)) we obtain $\frac{\partial I_{1}}{\partial r_{1}}\left(r_{1}, w\right)>0$, so the graph of $I_{1}(., w)$ is strictly increasing. Moreover as $\frac{\partial I_{1}}{\partial r_{1}}\left(r_{1}, w\right)<\frac{\pi q}{w}$ it follows that the graph of $I_{1}(., w)$ is below of the straight line $\frac{\pi q}{w} r_{1}$. Hence in order that the equation $h_{1}=0$ has solutions in $r_{1}$ with $r_{1}>w$, we have that $b_{1}\left(a_{11}+a_{22}\right)<0$.

(iii) $\lim _{r_{1} \rightarrow \infty} I_{1}(r, w)=4 q, I_{1}(., w):(0, \infty) \rightarrow(0,4 q)$ and $I_{1}$ is a $C^{1}-$ diffeomorphism.

In the same way we can see that for each $w$ fixed $I_{2 k}\left(r_{1}, w\right), k=2, \ldots, n$ are $C^{1}$-diffeomorphisms. This implies that the averaging function (17) is $C^{1}$

From (i)-(iii) we have that there exists in the interval $(w, \infty)$ at most one point of intersection between the graph of $I_{1}(., w)$ and the straight line through the origin $-\frac{\pi q\left(a_{11}+a_{22}\right)}{b_{1}} r_{1}$. Moreover this intersection point exists if and only if

$$
\left.0<-\frac{\left(a_{11}+a_{22}\right)}{b_{1}} w<1 \text { (see Figure } 1\right) .
$$

Consequently equation $h_{1}=0$ has exactly one solution in $r_{1}>w$ if we take $a_{11}, a_{22}$ and $b_{1}$ satisfying condition (18).

Denote this solution by $r_{1}^{*}$. Substituting it in the others equations of (17) for $k=\{2, \ldots, n\}$ we obtain

$$
\begin{aligned}
& \alpha_{k 1} r_{k}+\alpha_{k 2} u_{k}+\alpha_{k 3} v_{k}=0, \\
& \beta_{k}+\frac{1}{r_{k}}\left(-\alpha_{k 3} u_{k}+\alpha_{k 2} v_{k}\right)=0, \\
& u_{k}^{2}+v_{k}^{2}=1,
\end{aligned}
$$




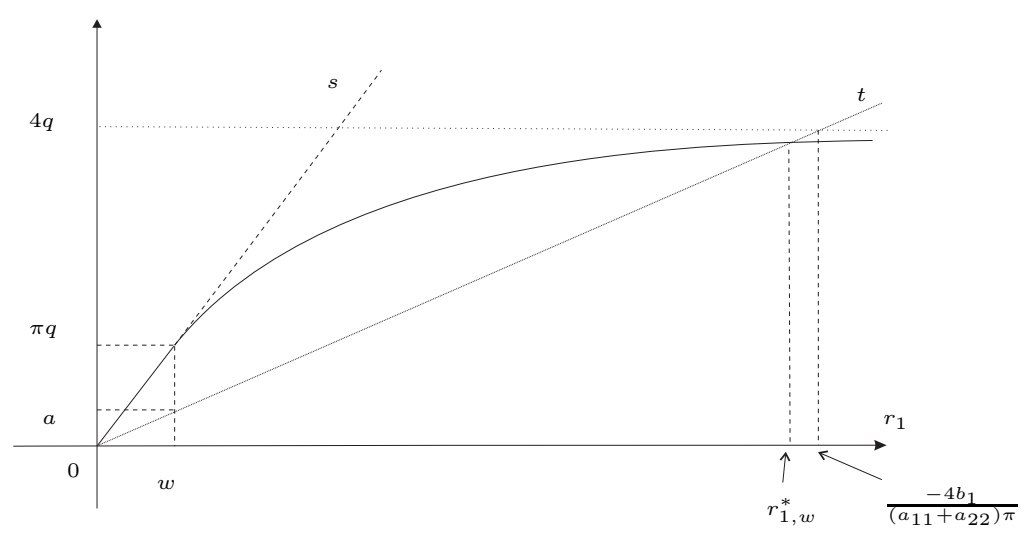

FiguRE 1. The graph of the function $I_{1}(., w)$ and the straight line $t$ given by equation $-\frac{\pi q\left(a_{11}+a_{22}\right)}{b_{1}} r_{1}$. We denote by $s$ the straight line $\frac{\pi q}{w} r_{1}$ and $a=-\frac{\pi q\left(a_{11}+a_{22}\right)}{b_{1}} w$.

where

$$
\begin{gathered}
\alpha_{k 1}=\pi q\left(a_{2 k-1,2 k-1}+a_{2 k, 2 k}\right), \quad \alpha_{k 2}=b_{2 k-1} I_{2 k}\left(r_{1}^{*}, w\right), \quad \alpha_{k 3}=b_{2 k} I_{2 k}\left(r_{1}^{*}, w\right), \\
\beta_{k}=\pi q\left(a_{2 k, 2 k-1}-a_{2 k-1,2 k}+a_{k} a_{2,1}-a_{k} a_{1,2}\right)+a_{k} b_{2} I_{1}\left(r_{1}^{*}, w\right) / r_{1}^{*},
\end{gathered}
$$

and

$$
u_{k}=\cos \theta_{k}, \quad v_{k}=\sin \theta_{k} .
$$

Note that

$$
\left|\begin{array}{cc}
\alpha_{k 2} & \alpha_{k 3} \\
-\alpha_{k 3} & \alpha_{k 2}
\end{array}\right|=\left(b_{2 k-1}^{2}+b_{2 k}^{2}\right) I_{2 k}\left(r_{1}^{*}, w\right)^{2} .
$$

So system (19) for each $k=2, \ldots, n$ has a unique solution for suitable election of the parameters $b_{2 k-1}, b_{2 k}, a_{1,2}, a_{2,1}, a_{2 k-1,2 k-1}, a_{2 k, 2 k}, a_{2 k, 2 k-1}$ and $a_{2 k-1,2 k}$. Hence for $\prod_{k=2}^{n}\left(b_{2 k-1}^{2}+b_{2 k}^{2}\right) \neq 0$ at most one limit cycle can bifurcate from the periodic orbits of the linear differential system (7) when this system is perturbed in the form (5). Moreover there are systems for which a such limit cycle exists. This proves Theorem 1.

Proof of Corollary 2. Let $\sum=\left\{x_{1}=0\right\}$ be a section for the flow of (1) and (5). Define $P_{o}: \sum \rightarrow \sum$ and $P_{w}: \sum \rightarrow \sum$ the first return map associated to (1) and (5) respectively.

Note that all the maps $P_{o}$ and $P_{w}$ for $w>0$ are analytic, $P_{o}$ is a composition of two analytic functions and $P_{w}$ is the composition of four analytic functions. Moreover, $\lim _{w \rightarrow 0} P_{w}=P_{o}$.

Now, from Theorem 1 we have that for each $w>0 P_{w}$ has at most a unique fixed point $x_{w}^{*} \in \sum$, with $x_{w}^{*} \neq 0$.

In the necessary condition for the existence of a such limit cycle (condition (18)) fixed $w \neq 0$, there exists a unique $r_{1, w}^{*}>w$ that satisfies the first equation of (17). 
This point is the point of intersection between the graph of the function $I_{1}(., w)$ and the straight line $-\frac{\pi q\left(a_{11}+a_{22}\right)}{b_{1}} r_{1}$.

Now note that for each $r_{1}>w$ we have

(a) $\frac{\partial I_{1}}{\partial w}=\frac{2 q}{w^{2}}\left(\frac{\sqrt{r_{1}^{2}-w^{2}}}{r_{1}} w-r_{1} \operatorname{arccsc}\left(\frac{r_{1}}{w}\right)\right)$ with $\lim _{r_{1} \searrow w} \frac{\partial I_{1}}{\partial w}\left(r_{1}, w\right)=-\frac{\pi q}{w}$ and $\lim _{r_{1} \rightarrow \infty} \frac{\partial I_{1}}{\partial w}\left(r_{1}, w\right)=0$.

(b) $\frac{\partial^{2} I_{1}}{\partial w^{2}}\left(r_{1}, w\right)=\frac{4 q r_{1}}{w^{2}}\left(\operatorname{arccsc}\left(\frac{r_{1}}{w}\right)-\frac{w}{\sqrt{r_{1}^{2}-w^{2}}}\right)<0$ for all $r_{1}>w$.

In fact, taking $f\left(r_{1}, w\right)=\operatorname{arccsc}\left(\frac{r_{1}}{w}\right)-\frac{w}{r_{1}^{2}-w^{2}}$ we have

Moreover

$$
\lim _{r_{1} \searrow w} f\left(r_{1}, w\right)=-\infty \quad \text { and } \quad \lim _{r_{1} \rightarrow \infty} f\left(r_{1}, w\right)=0 .
$$

$$
\frac{\partial f}{\partial r_{1}}\left(r_{1}, w\right)=-\frac{w^{3}}{r_{1}\left(r_{1}^{2}-w^{2}\right)^{3 / 2}}<0 .
$$

So $f\left(r_{1}, w\right)<0$ for $r_{1}>w$. This proves the assertion (b).

Now from (a) and (b) we obtain $\frac{\partial I_{1}}{\partial w}\left(r_{1}, w\right)<0$. So for $r_{1}$ fixed with $r_{1}>w$ we have $I_{1}\left(r_{1},.\right)$ decreasing with $w$ (see figure 2). This implies that if $w_{1}>w_{2}$ then $r_{1, w_{1}}^{*}<r_{1, w_{2}}^{*}$. Moreover an upper bounded of $r_{1, w}^{*}$ for $k=1,2$ is $\frac{-4 b_{1}}{\left(a_{11}+a_{22}\right) \pi}$. This implies that $\lim _{w \rightarrow 0} r_{1, w}^{*}=r_{1}^{*}$ exists and

$$
0<w<r_{1}^{*} \leq-\frac{4 b_{1}}{\left(a_{11}+a_{22}\right) \pi} .
$$

The existence of the limits of $r_{k, w}^{*}$ and $\theta_{k, w}^{*}, k=2, \ldots, 3$ when $w \rightarrow 0$ follow directly from the fact that these values are solutions of system (19) and they depend only on the parameters $a_{i j}, 1 \leq i, j \leq 2 n, b_{l}, l=1, \ldots, 2 n$ (fixed for a given one parameter family of system parameterized by $w$ ) and on $r_{1, w}^{*}$. So we have $\lim _{w \rightarrow 0}\left(r_{1, w}^{*}, \ldots, r_{n, w}^{*}, \theta_{2, w}^{*}, \ldots, \theta_{n, w}^{*}\right)=\left(r_{1}^{*}, \ldots, r_{n}^{*}, \theta_{2}^{*}, \ldots, \theta_{n}^{*}\right) \neq(0, \ldots, 0)$.

From the above arguments it follows that the fixed point $\left(r_{1, w}^{*}, \ldots, r_{n, w}^{*}, \theta_{2, w}^{*}, \ldots\right.$, $\left.\theta_{n, w}^{*}\right)$ of the averaging equation associated to the fixed point $x_{w}^{*}$ of $P_{w}$ have a non-zero limit when $w \rightarrow 0$. This implies that $x_{w}^{*} \rightarrow x^{*}$ and $x^{*} \neq 0$. Now as $\lim _{w \rightarrow 0} P_{w}\left(x_{w}^{*}\right)=P_{0}\left(x^{*}\right)$ we obtain $P_{0}\left(x^{*}\right)=x^{*}$. This concludes the proof of the corollary.

Remark 11. We observe that given a discontinuous vector field (11) with $w=0$ satisfying the condition $\left(a_{11}+a_{22}\right) b_{1}<0$ the necessary condition (18) for the existence of limit cycle for the continuous vector field can always be satisfied. In fact for this we have just to take $w$ sufficiently small.

\section{Proof of Theorem 3}

Lemma 12. Doing the change of coordinates from $\left(x_{1}, x_{2}, \ldots, x_{2 n-1}, x_{2 n}\right)$ to $\left(r_{1}, \ldots\right.$, $\left.r_{n}, \theta_{1}, \ldots \theta_{n}\right)$ where

$$
x_{2 k-1}=r_{k} \cos \theta_{k}, \quad x_{2 k}=r_{k} \sin \theta_{k}, \quad k=1, \ldots, n,
$$




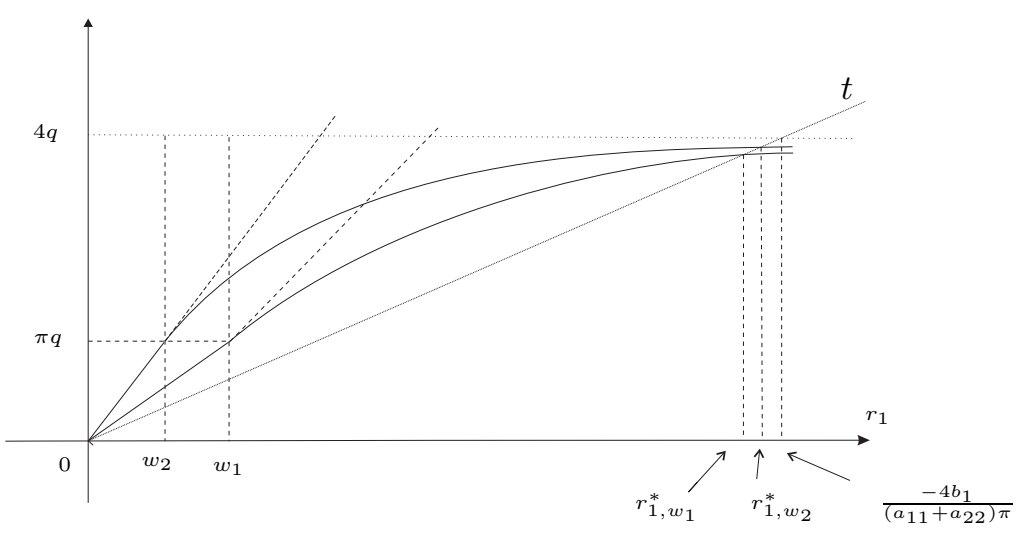

Figure 2. The graph of the function $I_{1}(., w)$ for two different values of $w$. Here $t$ denotes the straight line $-\frac{\pi q\left(a_{11}+a_{22}\right)}{b_{1}} r_{1}$.

and considering $\theta_{1}$ as the new time, system (11) can be written as

$$
\begin{array}{ll}
\frac{d r_{k}}{d \theta_{1}}=\varepsilon M_{1}\left(\theta_{1}, r_{1}, \ldots, r_{n}, \theta_{2}, \ldots \theta_{n}\right)+O\left(\varepsilon^{2}\right) & k=1, \ldots, n-1 \\
\frac{d r_{n}}{d \theta_{1}}=\varepsilon M_{n}\left(\theta_{1}, r_{1}, \ldots, r_{n}, \theta_{2}, \ldots \theta_{n}\right)+O\left(\varepsilon^{2}\right), & \\
\frac{d \theta_{l}}{d \theta_{1}}=a_{l}+O(\varepsilon) & l=2, \ldots, n,
\end{array}
$$

where

$$
\begin{aligned}
& M_{k}=r_{k+1} \cos \left(\theta_{k+1}-\theta_{k}\right)+\cos \theta_{k} L_{2 k-1}+\sin \theta_{k} L_{2 k}, \quad k=1, \ldots, n-1 \\
& M_{n}=\cos \theta_{n} L_{2 n-1}+\sin \theta_{n} L_{2 n} .
\end{aligned}
$$

The functions $L_{l}$ for $l=1, \cdots 2 n$ are given in Appendix 2.

Proof. The proof is similar to the proof of Lemma 9.

Observe that if $\left(r_{1}\left(\theta_{1}\right), \ldots, r_{n}\left(\theta_{1}\right), \theta_{2}\left(\theta_{1}\right), \ldots, \theta_{n}\left(\theta_{1}\right)\right)$ is a solution of $(20)$, then we have

$$
\theta_{l}\left(\theta_{1}\right)=a_{l} \theta_{1}+\theta_{l}^{o}+O(\varepsilon), \quad l=2, \ldots, n .
$$

Substituting these expressions in system (20) we obtain

$$
\begin{aligned}
& \frac{d r_{k}}{d \theta_{1}}=\varepsilon M_{1}\left(\theta_{1}, r_{1}, \ldots, r_{n}, a_{2} \theta_{1}+\theta_{2}^{o}, \ldots, a_{n} \theta_{1}+\theta_{n}^{o}\right)+O\left(\varepsilon^{2}\right), \\
& \frac{d r_{n}}{d \theta_{1}}=\varepsilon M_{n}\left(\theta_{1}, r_{1}, \ldots, r_{n}, a_{2} \theta_{1}+\theta_{2}^{o}, \ldots, a_{n} \theta_{1}+\theta_{n}^{o}\right)+O\left(\varepsilon^{2}\right),
\end{aligned}
$$

where $k=1, \ldots, n-1$.

Note that for fixed $\left(\theta_{2}^{o}, \ldots, \theta_{n}^{o}\right)$ any zero of the averaging system associated to system (21) corresponds to a limit cycle of (20). Moreover if the zero is persistent for $\left(\theta_{2}^{o}, \ldots, \theta_{n}^{o}\right) \in\left[\gamma_{21}, \gamma_{22}\right] \times \ldots \times\left[\gamma_{n 1}, \gamma_{n 2}\right]$ so system (11) has an invariant cylinder homeomorphic to $\mathbb{R} \times\left(\mathbb{S}^{1}\right)^{n-1}$ fulfilled of periodic orbits because all the $a_{l} \in \mathbb{Q}$ for $l=2, \cdots, n$. So all the periodic orbits have period $2 \pi q$. Here in order to find this invariant cylinders we have to study the averaging system associated to system (21). 
In this case the averaging function is given by $g\left(r_{1}, \cdots, r_{n}, \theta_{2}, \cdots, \theta_{n}\right)=\left(u_{1}, \cdots\right.$, $u_{n}$ ) where

$$
u_{k}\left(r_{1}, \ldots, r_{n}, \theta_{2}, \ldots, \theta_{n}\right)=\int_{0}^{2 \pi q} M_{k}\left(\theta_{1}, r_{1}, \ldots, r_{n}, a_{2} \theta_{1}+\theta_{2}^{o}, \ldots a_{n} \theta_{1}+\theta_{n}^{o}\right) d \theta_{1},
$$

for $k=1, \cdots, n$.

Lemma 13. The system obtained from (21) by the averaging function $g$ is given by

$$
\begin{aligned}
& u_{1}=\pi q r_{1}\left(a_{11}+a_{22}\right)+b_{1} I_{1}\left(r_{1}, w\right)=0, \\
& u_{k}=\pi q r_{k}\left(a_{2 k-1,2 k-1}+a_{2 k, 2 k}\right)+\left(b_{2 k-1} \cos \theta_{k}^{o}+b_{2 k} \sin \theta_{k}^{o}\right) I_{2 k}\left(r_{1}, w\right)=0,
\end{aligned}
$$

where $k=2, \ldots, n$.

Proof. The proof of this lemma is similar to the proof of Lemma 10 and it will be omitted.

With Lemma 22 we are in position to present the proof of Theorem 3

Proof of Theorem 3. As in the proof of Theorem 1 we will just find isolated solutions for the case $r_{1}>w$.

Note that the first equation of system (22) is exactly the first equation of system (17). So we can find a unique solution for the first equation provided that $b_{1}\left(a_{11}+\right.$ $\left.a_{22}\right)<0$. Substituting this solution $r_{1}^{*}$ into the others equations of (22) and solving then with respect to the variables $r_{k}$ for $k=2, \ldots, n$ we obtain

$$
r_{k}^{*}=-\frac{b_{2 k-1} \cos \theta_{k}^{o}+b_{2 k} \sin \theta_{k}^{o}}{\pi q\left(a_{2 k-1,2 k-1}+a_{2 k, 2 k}\right)} I_{2 k}\left(r_{1}^{*}, w\right) .
$$

Now note that these expressions provide positive solutions if $a_{2 k-1,2 k-1}+a_{2 k, 2 k} \neq$ $0, b_{2 k-1}^{2}+b_{2 k}^{2} \neq 0$ for $k=2, \ldots, n$ and for $\theta_{k}^{o}$ chosen in a convenient way.

Moreover, for each $k=2, \ldots, n$, given $\theta_{k}^{o}$ satisfying equation (23), it is possible to vary the angle $\theta_{k}^{o}$ in an interval in such a way that the solution obtained is locally persistent. This implies the existence of an invariant cylinder for system (11) homeomorphic to $\mathbb{R} \times\left(\mathbb{S}^{1}\right)^{n-1}$.

Proof of Corollary 4. The proof is similar to the proof of Corollary 2.

\section{EXAMPLE}

Consider the following differential system in $\mathbb{R}^{4}$

$$
\begin{aligned}
& \dot{x}_{1}=-x_{2}+\varepsilon\left(x_{1}+x_{3}-\varphi_{0}\left(x_{1}\right)\right), \\
& \dot{x}_{2}=x_{1}+\varepsilon x_{4}, \\
& \dot{x}_{3}=-2 x_{4}+\varepsilon x_{3}, \\
& \dot{x}_{4}=2 x_{3}+\varepsilon \varphi_{0}\left(x_{1}\right) .
\end{aligned}
$$

For this particular system the matrix $\bar{A}=\left(a_{i j}\right)$ and the vector $\bar{b}=\left(b_{l}\right)^{T}$ of Lemma 8 have $a_{11}=1=a_{33}$ and all the other entries zero, and $b_{1}=-1, b_{2}=$ $b_{3}=0, \quad b_{4}=1$. Moreover the entry $a_{2}$ of the Jordan matrix $A_{1}$ of Lemma 8 is $a_{2}=2$ and the integer $q$ here is $q=1$. So considering $w \leq 0.1$ we obtain

$$
0<-\frac{\left(a_{11}+a_{33}\right)}{b_{1}} w=w \leq 0.1<1,
$$


and the equation $h_{1}=0$ of (17) has a unique solution $r_{1}^{*}>0.1$. In fact we can see that $r_{1}^{*} \approx 1.27193$. Moreover substituting this solution in the other two equations of (17) we obtain

$$
\pi r_{2}+I_{4}^{*} v_{2}=0, \quad-I_{4}^{*} u_{2}=0
$$

where $I_{4}^{*}=2 J_{2}\left(r_{1}^{*}, w\right) \approx-8.4<0, u_{2}=\cos \theta_{2}$ and $v_{2}=\sin \theta_{2}$.

From this system we obtain $v_{1}=-\frac{\pi r_{2}}{I_{4}^{*}}$ and $u_{2}=0$. This implies $\theta_{2}^{*}=\pi / 2$, and from the fact that $u_{2}^{2}+v_{2}^{2}=1$ we obtain $r_{2}^{*}=-\frac{I_{4}^{*}}{\pi}$. So the averaging function $f$ given by (17) has $\left(r_{1}^{*},-I_{4}^{*} / \pi, \pi / 2\right)$ as a unique zero. Moreover the eigenvalues of $D f\left(r_{1}^{*},-I_{4}^{*} / \pi, \pi / 2\right)$ are all positive real numbers. This implies that system (24) has a unique limit cycle up to first order in the displacement function.

In the same way we can show that system (24) admits an invariant cylinder homeomorphic to $\mathbb{R} \times \mathbb{S}^{1}$.

\section{Appendix 1}

Here we provide the explicit expressions of the functions $F_{l}$ for $l=1, \cdots, 2 n$ :

$$
\begin{aligned}
F_{1}= & a_{1,1} r_{1} \cos \theta_{1}+\sum_{j=2}^{n} a_{1,2 j-1} r_{j} \cos \left(a_{j} \theta_{1}+\theta_{j}\right)+a_{1,2} r_{1} \sin \theta_{1} \\
& +\sum_{j=2}^{n} a_{1,2 j} r_{j} \sin \left(a_{j} \theta_{1}+\theta_{j}\right)+b_{1} \varphi_{w}\left(r_{1} \cos \theta_{1}\right), \\
F_{2}= & a_{21} r_{1} \cos \theta_{1}+\sum_{j=2}^{n} a_{2,2 j-1} r_{j} \cos \left(a_{j} \theta_{1}+\theta_{j}\right)+a_{2,2} r_{1} \sin \theta_{1} \\
& +\sum_{j=2}^{n} a_{2,2 j} r_{j} \sin \left(a_{j} \theta_{1}+\theta_{j}\right)+b_{2} \varphi_{w}\left(r_{1} \cos \theta_{1}\right), \\
F_{2 k-1}= & a_{2 k-1,1} r_{1} \cos \theta_{1}+\sum_{j=2}^{n} a_{2 k-1,2 j-1} r_{j} \cos \left(a_{j} \theta_{1}+\theta_{j}\right)+a_{2 k-1,2} r_{1} \sin \theta_{1} \\
& +\sum_{j=2}^{n} a_{2 k-1,2 j} r_{j} \sin \left(a_{j} \theta_{1}+\theta_{j}\right)+b_{2 k-1} \varphi_{w}\left(r_{1} \cos \theta_{1}\right), \\
F_{2 k}= & a_{2 k, 1} r_{1} \cos \theta_{1}+\sum_{j=2}^{n} a_{2 k, 2 j-1} r_{j} \cos \left(a_{j} \theta_{1}+\theta_{j}\right)+a_{2 k, 2} r_{1} \sin \theta_{1} \\
& +\sum_{j=2}^{n} a_{2 k, 2 j} r_{j} \sin \left(a_{j} \theta_{1}+\theta_{j}\right)+b_{2 k} \varphi_{w}\left(r_{1} \cos \theta_{1}\right), \\
F_{2 n-1}= & a_{2 n-1,1} r_{1} \cos \theta_{1}+\sum_{j=2}^{n} a_{2 n-1,2 j-1} r_{j} \cos \left(a_{j} \theta_{1}+\theta_{j}\right)+a_{2 n-1,2} r_{1} \sin \theta_{1} \\
& +\sum_{j=2}^{n} a_{2 n-1,2 j} r_{j} \sin \left(a_{j} \theta_{1}+\theta_{j}\right)+b_{2 n-1} \varphi_{w}\left(r_{1} \cos \theta_{1}\right), \\
& a_{2 n, 1} r_{1} \cos \theta_{1}+\sum_{j=2}^{n} a_{2 n, 2 j-1} r_{j} \cos \left(a_{j} \theta_{1}+\theta_{j}\right)+a_{2 n, 2} r_{1} \sin \theta_{1} \\
& +\sum_{j=2}^{n} a_{2 n, 2 j} r_{j} \sin \left(a_{j} \theta_{1}+\theta_{j}\right)+b_{2 n} \varphi_{w}\left(r_{1} \cos \theta_{1}\right),
\end{aligned}
$$

where $k=2, \cdots, n-1$.

\section{Appendix 2}

Here we provide the explicit expressions of the functions $L_{l}$ for $l=1, \cdots, 2 n$ :

$$
\begin{aligned}
L_{2 k-1} & =\sum_{j=1}^{n} a_{2 k-1,2 j} r_{j} \sin \theta_{j}+\sum_{j=1}^{n} a_{2 k-1,2 j-1} r_{j} \cos \theta_{j}+b_{2 k-1} \varphi_{w}\left(r_{1} \cos \theta_{1}\right), \\
L_{2 k} & =\sum_{j=1}^{n} a_{2 k, 2 j} r_{j} \sin \theta_{j}+\sum_{j=1}^{n} a_{2 k, 2 j-1} r_{j} \cos \theta_{j}+b_{2 k} \varphi_{w}\left(r_{1} \cos \theta_{1}\right) .
\end{aligned}
$$




\section{ACKNOWLEDGMENTS}

The first author is partially supported by CNPq grand number 200293/20109. The second author is partially supported by MCYT/FEDER grant MTM200803437, CIRIT grant number 2009SGR-410 and by ICREA Academia. Both authors are also supported by the joint project CAPES-MECD grant PHB-2009-0025-PC.

\section{REFERENCES}

[1] Buica, A. And Llibre, J. Averaging methods for finding periodic orbits via Brouwer degree. Bull. Sci. Math. 128 (2004), 7-22.

[2] Buica, A. AND Llibre, J. Bifurcation of limit cycles from a four-dimentional center in control systems. Int. J. of Bif. and Chaos. 15, 8 (2005), 2653-2662.

[3] Buica, A., Llibre, J., and Makarenkov, O. Asymptotic stability of periodic solutions for nonsmooth differential equations with application to the nonsmooth Van Der Pol oscillator. SIAM J. Math. Anal. 40, 6 (2009), 2478-2495.

[4] Cardin, P.T., De Carvalho, T. and Llibre, J. Bifurcation of limit cycle from a $n$ dimensional linear center inside a class of piecewise linear differential systems, preprint, 2010.

[5] Cardin, P.T., De Carvalho, T. and Llibre, J. Limit cycles of discontinuous piecewise linear differential systems, preprint, 2010.

[6] di Bernardo, M., Budd, C.J., Champneys, A.R. And Kowalczyk, P. Piecewise-smooth dynamical systems. Appl. Math. Sci. Series. 163, London, Springer, 2008.

[7] Llibre, J. And Makhlouf, A. Bifurcation of limit cycle from a 4-dimensional center in 1:n resonance. App. Math. and Computation. 215 (2009), 140-146.

[8] Lloyd, N.G. Degree Theory. Cambridge University Press, 1978.

[9] Utkin, V.I. Sliding Modes in Control Optimization. Berlin, Springer, 1992.

[10] Verhulst, F. Nonlinear Differencial Equations and Dynamical Systems. Universitext. 2nd edition. Springer, 1996.

1 Centro de Matematica ComputaÇão e CogniÇão. Universidade Federal do ABC, 09210-170. SANTO Andre. S.P. BRAZIL

E-mail address: mauricio.lima@ufabc.edu.br

2 Departament de Matemàtiques. Universitat Autònoma de Barcelona, 08193 Bellaterra. Barcelona, Catalonia, Spain

E-mail address: jllibre@mat.uab.cat 